\title{
The Loewner equation: maps and shapes
}

\author{
Ilya A. Gruzberg and Leo P. Kadanoff \\ The James Franck Institute, The University of Chicago \\ 5640 S. Ellis Avenue, Chicago, Il 60637 USA \\ e-mail: gruzberg@uchicago.edu and l-kadanoff@uchicago.edu
}

(Dated: September 10, 2003)

The most exciting concepts in theoretical physics are those that relate algebraic properties to geometrical ones. Of course, the outstanding example of this is the geometric meaning of the equations of general relativity, and their realization in the shapes of possible universes and in black holes. Other examples abound including the patterns of the paths of Brownian motion, the forms of percolating clusters, the shapes of snowflakes and phase boundaries, and the beautiful fingers of interpenetrating fluids and of dendrites.

Especially beautiful and important patterns are seen in the case in which these structures can be very large and fractal, as in a long random walk or in the critical phenomena which occur near higher order phase transitions. More than a century of effort has gone into studying the problems posed by these objects. Much of it has been devoted to figures in two dimensions. These shapes are varied enough to be interesting, but relatively easy to characterize and study.

In the last three years, new insights have permitted unexpected progress in the study of fractal shapes in two dimensions. A new approach has arisen through analytic function theory and probability theory, and given a new way of calculating fractal shapes in critical phenomena, and in other problems like diffusion limited aggregation (DLA), the theory of random walks, and of percolation.

\section{CONFORMAL MAPS}

It all starts with the Riemann mapping theorem which gives a method for characterizing shapes by using the theory of analytic functions of a complex variable. High school students know how to characterize a point $(x, y)$ in two-dimensional space by a complex number $z=x+i y$. With a little more thought they can understand how a region $\mathcal{D}$ in the $z$-plane that region might be mapped into another region $\mathcal{R}$ of the $w$-plane by the function $w=g(z)$.

More advanced analysis considers the case in which $g$ is a function which is analytic and univalent within $\mathcal{D}$. The last property means that different points in $\mathcal{D}$ have different images under the action of $g$ (or that $g$ does not "glue" points together). In this case it follows that the derivative of $g(z)$ does not vanish within the region $\mathcal{D}$. Then the mapping is called conformal, and it takes the boundary of $\mathcal{D}$ into the boundary of $\mathcal{R}$. Thus an analysis of functions of complex variables automatically connects to a theory of the shapes of regions and curves in two
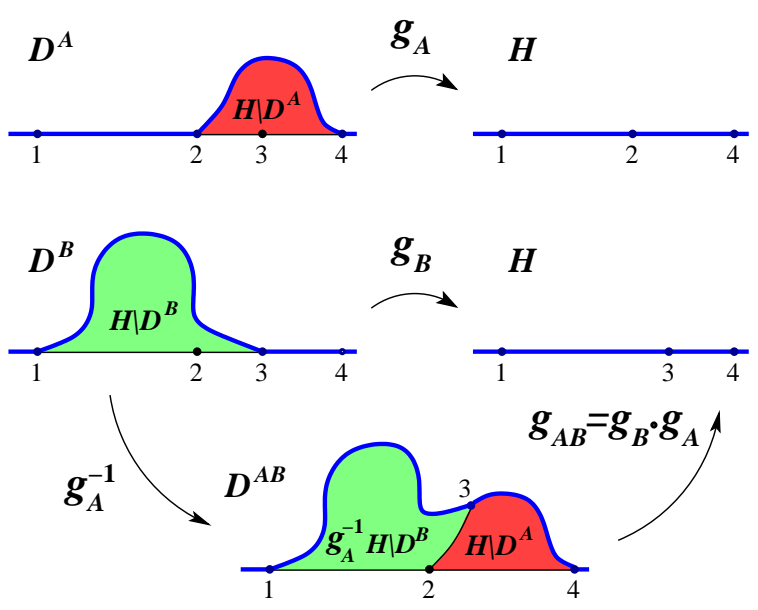

FIG. 1: Illustration for composition of conformal maps described in the text. The notation $\mathcal{H} \backslash \mathcal{D}^{A}$ means the complement of $\mathcal{D}^{A}$ in $\mathcal{H}$. Correspondence of points on the real axis and the boundary of $\mathcal{D}^{A B}$ is shown.

dimensions.

The Riemann mapping theorem states that any simply connected region whose boundary has more then one point can be transformed in an essentially unique manner into any other such region by a conformal transformation. We shall use the theorem to convert a region of interest, for example a region contained in the upper half plane, into a reference region, for example the entire upper half plane, conventionally written as $\mathcal{H}$. To get all the theorems we want, we shall need a few more constraints: our regions are simply connected and contain the point at infinity. The mappings take the form

$$
g(z) \rightarrow z+\frac{a}{z}+O\left(z^{-2}\right)
$$

as $z$ goes to infinity.

The conformal mapping functions have a very important composition property. Let the functions $g_{A}$ and $g_{B}$ respectively map the regions $\mathcal{D}^{A}$ and $\mathcal{D}^{B}$ into the upper half plane. Then the composition of the two $g_{A B}(z)=$ $g_{B}\left(g_{A}(z)\right)$ maps a region $\mathcal{D}^{A B}$ which is contained within $\mathcal{D}^{A}$ into the upper half plane (in the set-theoretic notation $\mathcal{D}^{A B}=\mathcal{D}^{A} \backslash g_{A}^{-1}\left(\mathcal{H} \backslash \mathcal{D}^{B}\right)$, see Fig. 1 for an illustration). Another composition, giving $g=g_{C} \cdot g_{B} \cdot g_{A}$ maps a subset of $\mathcal{D}^{A B}$ into the reference region. Successive iterations gives us successively "smaller" regions. 


\section{LOEWNER EQUATION}

To study this situation, Loewner defined families of maps, $g_{t}$, defined by a real parameter $t$, which we might call time, and by a real "driving" function $\xi(t)$. The Loewner equation

$$
\frac{d}{d t} g_{t}(z)=\frac{2}{g_{t}(z)-\xi(t)}, \quad g_{t=0}(z)=z
$$

defines a family of conformal maps $w=g_{t}(z)$ which take a subregion of the upper half $z$-plane into the upper half $w$-plane. The equation (2) is written for a somewhat arbitrary (but convenient) choice of the time variable, such that the map $g_{t}(z)$ satisfies the asymptotic condition (11) with $a=2 t$.

At time zero, $g_{t=0}$ is the identity map which maps $\mathcal{H}$ into itself. The boundary curve is the real axis and that too maps into itself. At each subsequent time $t, g_{t}(z)$ defines a new mapping-region $\mathcal{D}_{t}$ which maps into $\mathcal{H}$. Equally, the boundary of this region maps into the real axis of the $w$-plane.

The most important properties of $g_{t}(z)$ arise from the composition properties of conformal maps. Consider two maps $g_{t}^{A}(z)$ and $g_{t}^{B}(z)$ generated respectively by the forcing functions $\xi^{A}(t)$ and $\xi^{B}(t)$ which exist in the respectively exist in intervals $\left[0, t_{A}\right]$ and $\left[0, t_{B}\right]$. Now, write $g_{A}=g_{t_{A}}^{A}(z)$ and $g_{B}=g_{t_{B}}^{B}(z)$ for the maps generated by Eq. (2) for each forcing and consider the composite forcing:

$$
\begin{aligned}
\xi(t) & =\xi^{A}(t) \text { for }\left(0<t<t_{A}\right) \\
& =\xi^{B}\left(t-t_{A}\right) \text { for }\left(t_{A}<t<t_{A}+t_{B}\right) .
\end{aligned}
$$

Then the composition rule indicates that the composite forcing generates a map $g_{t}$ such that by the time $t_{A}+t_{B}$ it is simply

$$
g_{t_{A}+t_{B}}(z)=g_{B}\left(g_{A}(z)\right) .
$$

Indeed, fix $z$ and calculate $g_{t}(z)$ for $0<t \leqslant t_{A}$ from Eq. (2) using the initial condition $g_{t=0}(z)=z$. Then repeat the calculation for $t_{A}<t \leqslant t_{B}$ with the initial condition $g_{t=t_{A}}(z)=g_{A}(z)$. The result at $t=t_{B}$ is precisely described by the composition in the Eq. (4).

An immediate consequence of this composition rule is that the mapping sets $\mathcal{D}_{t}$ continually get "smaller" as $t$ gets larger. More properly stated if $s$ is greater than $t$ then $\mathcal{D}_{t}$ contains $\mathcal{D}_{s}$.

In some sense, we can watch the mapping region get smaller. This happens at time $t$ when some points $z_{c}(t)$ pass out of the domain of analyticity of $g_{t}$. From the Loewner equation that will happen as the denominator in equation (2) passes through zero or at the points which obey

$$
g_{t}\left(z_{c}(t)\right)=\xi(t) .
$$

Since $\xi(t)$ is real, that is, always on the boundary of $\mathcal{H}$, its pre-image $z_{c}(t)$ always sits on the edge of the region
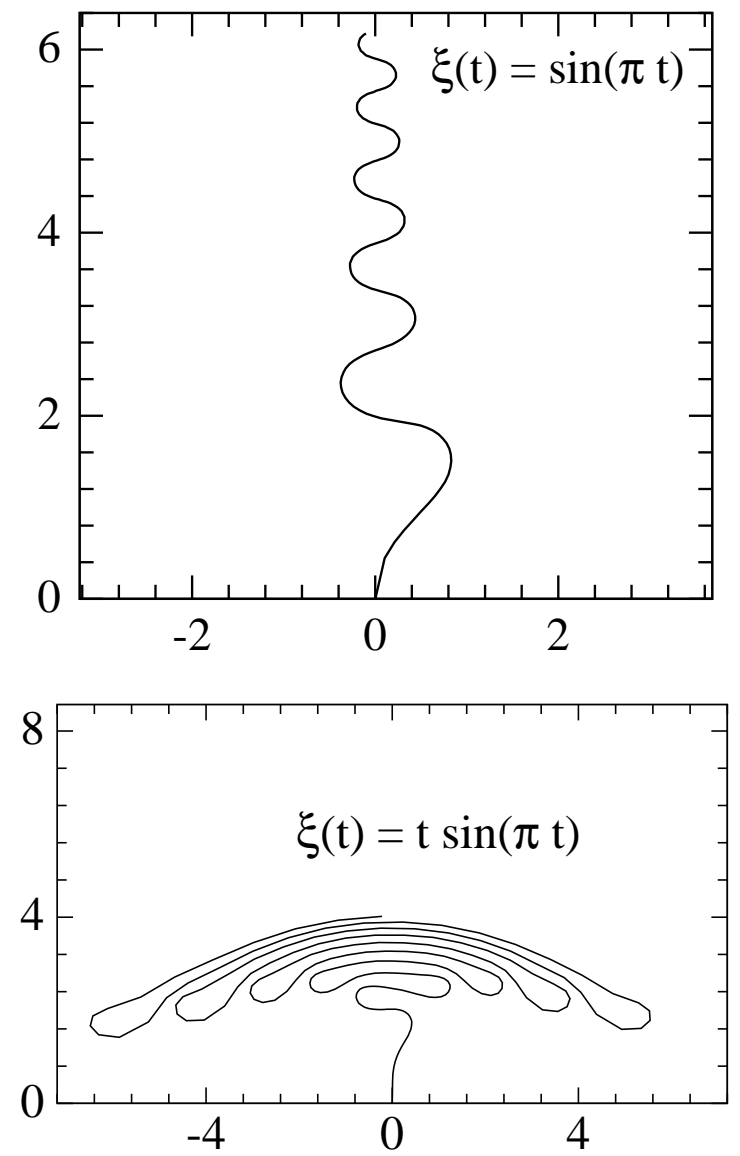

FIG. 2: The upper figure shows the trace for a periodic driving function $\xi(t)=\sin (t)$. The trace looks similar at different scales. The trace on the lower figure is driven by $\xi(t)=$ $t \sin (t)$.

$\mathcal{D}_{t}$ that is mapped to $\mathcal{H}$ by the function $g_{t}(z)$. If $\xi(t)$ is continuous, as time goes on the singularities trace out a continuous curve in $\mathcal{H}$, which we call simply the trace. The composition property implies that if a point is in the trace now, it will remain in it for all subsequent times. The trace is then a permanent path which shows where the singularities arise and what points have been removed from the mapping region.

The properties of the bounding curve composed of the trace and the real axis, are absolutely amazing:

- if $\xi(t)$ is smooth enough so that its derivative exists everywhere, the bounding curve never intersects itself.

- if $\xi(t)$ is periodic, the bounding curve is a selfsimilar object. See Figure 2 for an example.

- the curve can intersect itself at finite time only if $\xi(t)$ is sufficiently singular. The condition required 1] is that for some $t$

$$
\lim _{\tau \rightarrow 0^{+}}\left|\frac{\xi(t-\tau)-\xi(t)}{\tau^{0.5}}\right|
$$



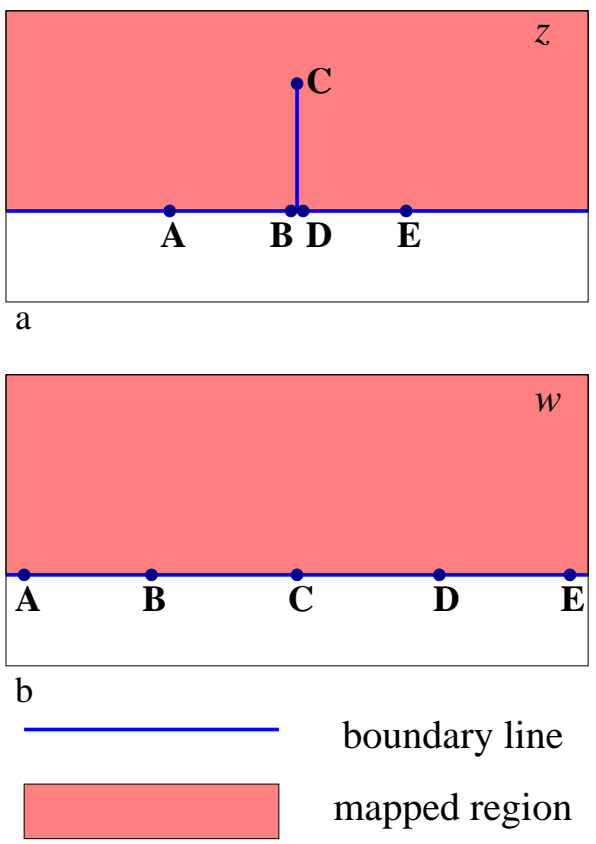

FIG. 3: This figure shows how a slit in the upper half of the $z$-plane (part a) maps into a linear boundary in the $w$-plane (part b). Notice that two neighboring points in the $z$-plane have images which are far apart in $w$.

goes to a value greater than 4 .

Thus the topological properties of the generated curve are directly, but non-trivially, related to the analytic properties of the forcing function $\xi(t)$.

To see what happens in a specific example choose $\xi(t)$ to be a constant, say $c$, independent of time. Then equation (2) has the solution:

$$
g_{t}(z)=c+\left((z-c)^{2}+4 t\right)^{1 / 2}
$$

As we can see in Figure 3 the singularity at time $t$ is at $z_{c}(t)=c+2 i t^{1 / 2}$. Thus, the trace is a straight line which extends from $z=c$ to $z=c+2 i t^{1 / 2}$.

More complex generating functions give much more complex boundary curves. For example, imagine that at some time, $\xi(t)$ has a discontinuous jump. The boundary then gains a new segment, see Figure 4 either having the new curve coming out of the horizontal base or having it branch from the old segment. On the other hand, if $\xi(t)$ varies sufficiently smoothly, the boundary curve retains the topology shown in Figure $4 \mathrm{k}$. It is a smooth curve which is extended further and further as time progresses, but never crosses itself. A few exact solutions of the Loewner equation were obtained in a recent paper [2].

\section{FROM LOEWNER TO CRITICAL PHENOMENA}

The years following Loewner's initial work showed many applications of complex analytic methods to the
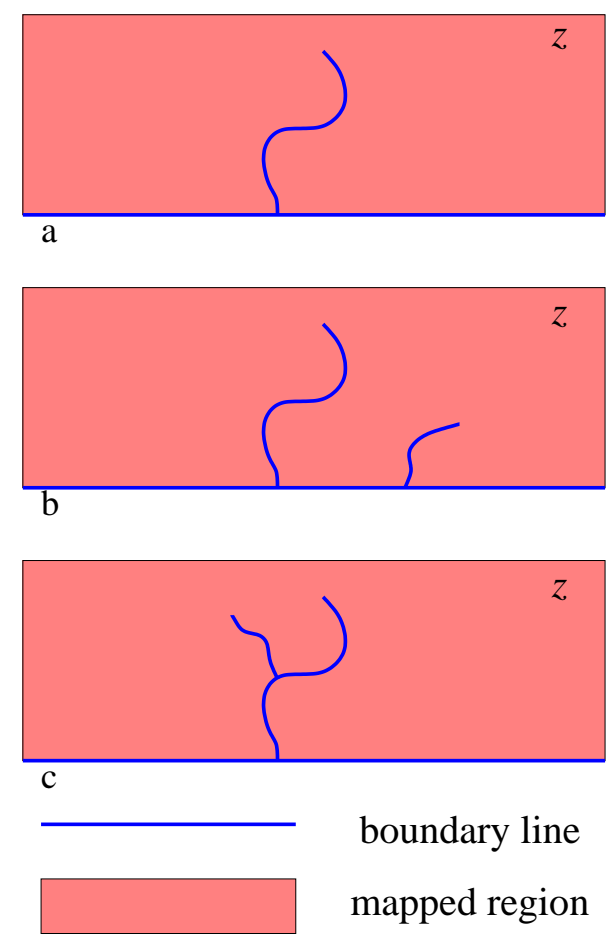

FIG. 4: Part a is a smooth curve produced by a differentiable forcing function. Parts $\mathrm{b}$ and $\mathrm{c}$ show different traces produced by a discontinuous $\xi(t)$.

study of problems involving fractal or quasi-fractal objects in the plane.

One major approach was reformulation by Hastings and Levitov [3] of the DLA model originally put forward by Witten and Sander [4]. The latter authors considered a step by step process in which an aggregate composed of many soot particles grew into a large, fractal object. A new tiny piece of soot would appear far away from the aggregate and undergo a random walk, ending when the piece touched the aggregate and stuck on. Iterations of this process made for a very large and interesting object. Hastings and Levitov represented the addition process by a conformal map from a circle to a circle with a bump upon it. When such process is iterated many times, the composition rule for conformal maps produced a continually growing object. Since the random walk and conformal function both obey the Laplace equation, DLA can be represented by choosing the addition point at random. Hence Hastings and Levitov constructed a stochastic procedure within the general class considered by Loewner. This reformulation might well have spurred people on to think about what would happen if one combined Loewner and stochasticity.

Another major use of analytic function theory for two dimensional fractals involved noticing that behavior near critical points had many invariance properties, including invariance under conformal transformations. According to the work of Polyakov [5] and others [ 6 , the scale invariance characteristic of behavior near critical points 
could be quite naturally generalized to include all nonlocal shear-free transformations 7] which then implied conformal invariance. A wide variety of critical problems were analyzed using conformal methods, but with a few exceptions the work was limited to the behavior of thermodynamic functions and of point operators like the magnetization. As noted above, conformal methods were also applied to such problems as Brownian motion, selfavoiding walks, percolation and DLA since these were all considered to be somehow close to critical phenomena. Indeed several of these problems were shown to be limiting cases of the critical models. But, in recent years, work on critical phenomena slowed down somewhat because it was felt that most of the leading problems had been investigated.

\section{SCHRAMM-LOEWNER EVOLUTION}

However, this view has proven wrong. The area of twodimensional (2D) critical phenomena has enjoyed a recent breakthrough. A radically new development, called the Schramm-Loewner Evolution or SLE (also previously called "stochastic Loewner evolution") 8, 9| has provided us new tools and new questions for criticality in 2D, and also provides us with a new interpretation of the traditional conformal field theory (CFT) approach.

Examples of systems described by SLE include familiar statistical models — Ising, Potts, $O(n)$ model, polymers, - as well as "geometric" critical phenomena like percolation, self-avoiding random walks, spanning trees and others. The new description focuses directly on nonlocal structures that characterize a given system, be it a boundary of an Ising or percolation cluster, or loops in the $O(n)$ model. This description uses the fact that all these non-local objects become random curves at a critical point in the continuum limit, and these curves may be precisely characterized by the stochastic dynamics which we shall describe in a moment.

This is an exciting development in that SLE complements the earlier approaches to problems in critical phenomena. It appears that questions that are difficult to pose and/or answer within CFT are easy and natural in the SLE framework, and vice versa. One of the challenges of the near future is to extend the overlap between the two approaches as far as possible and see whether they are really equivalent.

So what is SLE? It is simply the study of the Loewner equation with stochastic driving, specifically driving by a $\xi(t)$ which a Gaussian random variable, obeying the familiar Langevin equations of Brownian motion,

$$
\langle\dot{\xi}(t) \dot{\xi}(s)\rangle=\kappa \delta(t-s) .
$$

or equivalently in more integrated form

$$
\left\langle(\xi(t)-\xi(s))^{2}\right\rangle=\kappa|t-s| .
$$

Here $\kappa$ is a dimensionless constant whose value is very

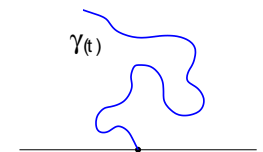

$0<\kappa \leqslant 4$
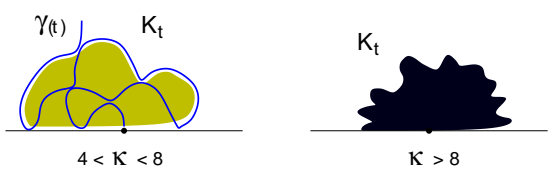

FIG. 5: Three different traces produced by SLE. This sketch is taken from Bauer and Bernard "Conformal field theories of stochastic Loewner evolutions", arXiv:hep-th/0210015

important determinant of the behavior. It is usual to refer to SLE at a particular value of $\kappa$ as $\mathrm{SLE}_{\kappa}$.

It was Schramm's idea 8] that one can use Loewner equation to describe conformally-invariant random curves if one chooses $\xi(t)$ to be a random function that satisfies certain conditions. First, $\xi(t)$ must be continuous with probability one. Secondly, to produce a conformally-invariant curve, the process $\xi(t)$ has to have independent identically distributed increments, since one can construct the required map $g_{t}(z)$ by iterations of some infinitesimal identically-distributed conformal maps. Further natural requirement of the invariance of $g_{t}(z)$ with respect to reflection $x+i y \rightarrow-x+i y$ makes the choice of $\xi(t)$ essentially unique: it can only be a scaled version of the Brownian motion without drift of Eq. (8).

We have already seen that if we chose $\xi(t)$ to be a smooth real-valued function, the solution $g_{t}(z)$ of Eq. (2) would give a conformal map from $\mathcal{H}$ cut along a segment traced out by a simple, non self-intersecting, curve $\gamma$. Similarly, in SLE $g$ gives a map from a $\mathcal{D}_{t}$ in $\mathcal{H}$ onto $\mathcal{H}$. This region is $\mathcal{H}$ with some part cut away by the singularities generated in $g$. The cut out part might be a simple curve which avoids the real line (for $0<\kappa \leqslant 4$ ), a self-intersecting one (for $4<\kappa<8$ ), or a filled in region (for $\kappa \geqslant 8$ ). For pictures of these possibilities see Figure 5] There are theorems and speculations saying that the trace of the cut-out singularities or the curves which surround the self-intersection trace, or the filled in region gives direct and useful information about the clusters and other geometrical objects formed in critical phenomena and other associated scale-invariant processes.

The theorems apply to percolation, the so-called looperased random walks, and the uniform spanning trees, which are related, correspondingly, to $\mathrm{SLE}_{6}, \mathrm{SLE}_{2}$, and $\mathrm{SLE}_{8}$. They state essentially that the ensemble of all the appropriate SLE objects is identical to the conformallyinvariant scaling limit of the critical cluster boundaries for percolation, or of paths — for loop-erased random walks and the space-filling curves winding along the uniform spanning trees. It is also known that if self-avoiding walks have a conformally-invariant continuum limit, this limit must be described by $\mathrm{SLE}_{8 / 3}$.

The corresponding critical phenomena statements (conjectures) are equally straightforward. They start by connecting each phase transition problem with a value of $\kappa$. For example, the standard critical Ising model is said to corresponds to $\kappa=3$. Various $\kappa$-values are similarly 
ascribed to the $O(n)$ model, the $q$-state Potts model (in which case $\sqrt{q}=-2 \cos (4 \pi / \kappa))$, . . each at their critical point. The the ensemble of traces or boundaries for the SLE process is argued to be identical with the ensemble of scaling-limit cluster boundaries for the critical problem. Cardy [10] has suggested that the correlation functions of boundary operators in critical phenomena could be explicitly calculated by using SLE methods. One of the first SLE-style calculations was due to Duplantier [11], who was interested in the ensemble of field strengths which would be realized in the neighborhood of a Potts model or Ising model cluster if that cluster were a charged conductor. He needed solutions to the Laplace equation in the exterior of the conductor, but any analytic function automatically provides such solutions. Hence the mapping functions $g$ would provide the necessary information. All three of these calculations, and many more are new results, unexpectedly available from SLE methods.

We shall not summarize the many important advances made with the aid of SLE methods, nor speculate about the like advances to come. Instead we simply say that this is a beautiful area of work, begun but not completed, where statistical scientists and analysts may hope to make further important advances. A bibliography on the subjects discussed in this paper is included in the appendix.

A review for physics audience on SLE and its relation to discrete models by Wouter Kager and Bernard Nienhuis is planned for a later issue of this journal.

\section{ACKNOWLEDGEMENTS}

This work was primarily supported by the NSF MRSEC Program under DMR-0213745. At various times the authors have had helpful interactions with Michel Bauer, Marko Kleine Berkenbusch, Denis Bernard, John Cardy, Isabelle Claus, Wouter Kager, Bernard Nienhuis, Ilia Rushkin, and Paul Wiegmann on subjects related to this note. Figure 2 was produced by Wouter Kager, who has kindly given us permission to use it. We thank Michel Bauer and Denis Bernard for permitting us to use their sketch in figure 5

\section{APPENDIX: REFERENCES ON SLE AND RELATED SUBJECTS.}

\section{History}

The Loewner equation first appeared in the paper

1. K. Löwner (C. Loewner), Untersuchungen über schlichte konforme Abbildungen des Einheitskreises, I. MAth. Ann. 89, 103, 1923.

The equation was proposed as a way of attacking the so called Bieberbach's conjecture. The conjecture was stated in 1916, and was finally proven in 1984 by de Branges. The proof relies heavily on the Loewner's method. There are several books with accounts of the Loewner's method and the proof of the conjecture. We list three:

2. L. V. Ahlfors, Conformal invariants: topics in geometric function theory, New York, McGraw-Hill, 1973.

3. P. L. Duren, Univalent functions, New York, Springer-Verlag, 1983.

4. Sheng Gong, The Bieberbach conjecture, Providence, R.I. American Mathematical Society: International Press, 1999.

\section{Pure math papers}

a. Reviews.

5. G. F. Lawler, Conformally invariant processes in the plane, a draft of a book available online at URL http://www.math. cornell.edu/ lawler/book.ps

6. G. F. Lawler, Conformally invariant processes in the plane, available online at URL http://www. math. cornell.edu/ lawler/papers.html

7. G. F. Lawler, Conformal invariance, universality, and the dimension of the Brownian frontier, Proceedings of the International Congress of Mathematicians, Vol. III, 63-72 (Higher Ed. Press, Beijing, 2002); arXiv: math.PR/0304369

8. G. Lawler, Introduction to the stochastic Loewner evolution, available online at URL http: //www. math. cornell.edu/ lawler/papers.html

9. O. Schramm, Scaling limits of random processes and the outer boundary of planar Brownian motion, Current developments in mathematics, 2000, 233-253 (Int. Press, Somerville, MA, 2001).

10. W. Werner, Critical exponents, conformal invariance and planar Brownian motion, arXiv: math.PR/0007042

11. W. Werner, Random planar curves and SchrammLoewner evolutions, arXiv: math.PR/0303354

12. W. Werner, Conformal restriction and related questions, arXiv: math.PR/0307353

\section{b. Research papers.}

The original paper where the SLE was introduced is

13. O. Schramm, Scaling limits of loop-erased random walks and uniform spanning trees, Israel J. Math., 118, 221-288, 2000; arXiv: math.PR/9904022

Many more papers followed.

14. R. O. Bauer, Loewner's equation in noncommutative probability, arXiv: math.PR/0208212.

15. R. O. Bauer, Discrete Loewner evolution, arXiv: math.PR/0303119

16. R. O. Bauer, Chordal Loewner families and univalent Cauchy transforms, arXiv: math.PR/0306130.

17. V. Beffara, On conformally invariant subsets of the planar Brownian curve, arXiv: math.PR/0105192 
18. V. Beffara, Hausdorff Dimensions for $S L E_{6}$, arXiv: math.PR/0204208

19. V. Beffara, The dimension of the SLE curves, arXiv: math.PR/0211322

20. L. Carleson and N. Makarov, Aggregation in the Plane and Loewners Equation, Commun. Math. Phys. 216, 583 (2001).

21. L. Carleson and N. Makarov, Laplacian path models, J. Anal. Math. 87, 103-150 (2002).

22. J. Dubedat, SLE and triangles, Electron. Comm. Probab. 8, 28-42 (2003); arXiv: math.PR/0212008.

23. J. Dubedat, Reflected planar Brownian motions, intertwining relations and crossing probabilities, arXiv: math.PR/0302250.

24. J. Dubedat, $S L E(\kappa, \rho)$ martingales and duality, arXiv: math.PR/0303128

25. J. Dubedat, Critical percolation in annuli and $S L E_{6}$, arXiv: math.PR/0306056

26. R. Friedrich, W. Werner, Conformal fields, restriction properties, degenerate representations and SLE. C. R. Math. Acad. Sci. Paris 335, 947-952 (2002); arXiv: math.PR/0209382.

27. R. Friedrich, W. Werner, Conformal restriction, highest-weight representations and SLE, arXiv: math.PR/0301018.

28. P. Kleban and D. Zagier, Crossing probabilities and modular forms, arXiv: math-ph/0209023.

29. G. F. Lawler, O. Schramm, W. Werner, Values of Brownian intersection exponents I: Half-plane exponents, Acta Math. 187, 237-273 (2001); arXiv: math.PR/9911084.

30. G. F. Lawler, O. Schramm, W. Werner, Values of Brownian intersection exponents II: Plane exponents, Acta Math. 187, 275-308 (2001); arXiv: math.PR/0003156

31. G. F. Lawler, O. Schramm, W. Werner, Values of Brownian intersection exponents III: Two sided exponents, Ann. Inst. H. Poincare Probab. Statist. 38, no 1, 109-123 (2002); arXiv: math.PR/0005294

32. G. F. Lawler, O. Schramm, W. Werner, Analyticity of intersection exponents for planar Brownian motion, Acta Math. 189, 179-201 (2002); arXiv: math.PR/0005295.

33. G. F. Lawler, O. Schramm, W. Werner, The dimension of the planar Brownian frontier is 4/3, Math. Res. Lett. 8, 401-411 (2001); arXiv: math.PR/0010165

34. G. F. Lawler, O. Schramm, W. Werner, Sharp estimates for Brownian non-intersection probabilities, in "In and out of equilibrium" (Mambucaba, 2000), 113131, Progr. Probab. 51 (Birkhuser Boston, Boston, MA, 2002); arXiv: math.PR/0101247

35. G. F. Lawler, O. Schramm, W. Werner, Onearm exponent for 2D critical percolation, Electr. J. Pobab. 7, no 2, 13 pp. (electronic) (2002); arXiv: math.PR/0108211.

36. G. F. Lawler, O. Schramm, W. Werner, Conformal invariance of planar loop-erased random walks and uniform spanning trees, Ann. Probab., to appear; arXiv: math.PR/0112234

37. G. F. Lawler, O. Schramm, W. Werner, On the scaling limit of planar self-avoiding walk, arXiv: math.PR/0204277

38. G. F. Lawler, O. Schramm, W. Werner, Conformal restriction: the chordal case, J. Amer. Math. Soc., to appear; arXiv: math.PR/0209343.

39. G. F. Lawler, W. Werner, Intersection exponents for planar Brownian motion, Ann. Probab. 27, 16011642.

40. G. F. Lawler, W. Werner, Universality for conformally invariant intersection exponents, J. European Math. Soc. 2, 291-328.

41. G. F. Lawler, W. Werner, The Brownian loop soup, arXiv: math.PR/0304419

42. D. Marshall and S. Rohde, The Loewner differential equation and slit mappings, available online at URL http://www. math. washington. edu/ rohde/

43. S. Rohde, O. Schramm, Basic properties of SLE, arXiv: math.PR/0106036

44. O. Schramm, A percolation formula, Electr. Comm. Probab. 6, 115-120 (2001); arXiv: math.PR/0107096

45. S. Smirnov, Critical percolation in the plane: conformal invariance, Cardys formula, scaling limits, C.R. Acad. Sci. Paris Sér. I Math. 333, 239-244 (2001).

46. S. Smirnov, Critical percolation in the plane. I. Conformal invariance and Cardys formula. II. Continuum scaling limit, available online at URL http://www.math.kth.se/ ${ }^{\sim}$ stas/papers/

47. S. Smirnov, W. Werner, Critical exponents for two-dimensional percolation, Math. Res. Lett. 8, 729744, 2001; arXiv: math.PR/0109120

48. W. Werner, Girsanov's transformation for $\operatorname{SLE}(\kappa, \rho)$ processes, intersection exponents and hiding exponents, arXiv: math.PR/0302115.

49. W. Werner, SLEs as boundaries of clusters of Brownian loops, arXiv: math.PR/0308164

\section{Physics and computational papers}

$$
\text { a. Reviews. }
$$

50. J. Cardy, Conformal invariance in percolation, self-avoiding walks and related problems, arXiv: cond-mat/0209638

51. B. Duplantier, Conformal fractal geometry and boundary quantum gravity, arXiv: math-ph/0303034.

\section{b. Research papers.}

52. M. Aizenman, B. Duplantier, and A. Aharony, Path-Crossing Exponents and the External Perimeter in 2D Percolation, Phys. Rev. Lett. 83, 1359 (1999); arXiv: cond-mat/9901018 
53. M. Bauer, D. Bernard, $\mathrm{SLE}_{k}$ growth processes and conformal field theories, Phys. Lett. B 543, 135138 (2002); arXiv: math-ph/0206028

54. M. Bauer, D. Bernard, Conformal field theories of stochastic Loewner evolutions, to appear in Commun. Math. Phys.; arXiv: hep-th/0210015

55. M. Bauer, D. Bernard, SLE martingales and the Virasoro algebra, Phys. Lett. B 557, 309-316 (2003); arXiv: hep-th/0301064.

56. M. Bauer, D. Bernard, Conformal transformations and the SLE partition function martingale, arXiv: math-ph/0305061

57. J. Cardy, Crossing formulae for critical percolation in an annulus, J. Phys. A: Math. Gen. 35, L565-L572 (2002); arXiv: math-ph/0208019

58. J. Cardy, Stochastic Loewner evolution and Dyson's circular ensembles, J. Phys. A: Math. Gen. 36, L379-L386 (2003); arXiv: math-ph/0301039.

59. B. Duplantier, Two-dimensional copolymers and exact conformal multifractality, Phys. Rev. Lett. 82, 880 (1999); arXiv: cond-mat/9812439

60. B. Duplantier, Harmonic measure exponents for two-dimensional percolation, Phys. Rev. Lett. 82, 3940 (1999); arXiv: cond-mat/9901008
61. B. Duplantier, Conformally invariant fractals and potential theory, Phys. Rev. Lett. 84, 1363 (2000); arXiv: cond-mat/9908314

62. B. Duplantier, Higher conformal multifractality, J. Stat. Phys. 110, 691-738 (2003); arXiv: cond-mat/0207743

63. B. Duplantier and I. A. Binder, Harmonic measure and winding of conformally invariant curves, Phys. Rev. Lett. 89, 264101 (2002); arXiv: cond-mat/0208045.

64. M. B. Hastings, Exact multifractal spectra for arbitrary laplacian random walks, Phys. Rev. Lett. 88, 055506 (2002); arXiv: cond-mat/0109304.

65. M. B. Hastings and L. S. Levitov, Laplacian growth as one-dimensional turbulence, Physica D 116, 244 (1998); arXiv: cond-mat/9607021

66. T. Kennedy, Monte Carlo tests of SLE Predictions for the 2D self-avoiding walk, arXiv: math.PR/0112246

67. T. Kennedy, Conformal invariance and stochastic Loewner evolution predictions for the 2D self-avoiding walk - Monte Carlo tests, arXiv: math.PR/0207231.

68. M. G. Stepanov and L. S. Levitov, Laplacian growth with separately controlled noise and anisotropy, Phys. Rev. E 63, 061102 (2001); arXiv: cond-mat/0005456
[1] As stated in the work of D. Marshall and S. Rohde (see "The Loewner differential equation and slit mappings", available online at URL http: //www . math. washington. edu/ ${ }^{\text {rohde/), }}$ behavior changes qualitatively when the coefficient in front of the singularity passes through a critical value. In a work in process 2], a few exact solutions of the Loewner evolution were obtained including one for a square root singularity. According to this paper the critical value is four.

[2] B. Nienhuis, W. Kager, and L. P. Kadanoff, in preparation.

[3] M. B. Hastings and L. S. Levitov, Physica D 116, 244 (1998); arXiv: cond-mat/9607021

[4] T. A. Witten and L. M. Sander, Phys. Rev. Lett. 47, 1400 (1981).
[5] A. M. Polyakov, JETP Letters 12, 381 (1970).

[6] A. A. Belavin, A. M. Polyakov, and A. B. Zamolodchikov, Nucl. Phys. B 241, 333 (1984).

[7] J. Cardy, Scaling and renormalization in statistical physics, Cambridge University Press, Cambridge, 1996.

[8] O. Schramm, Israel J. Math. 118, 221 (2000); arXiv: math.PR/9904022

[9] G. F. Lawler, O. Schramm and W. Werner, Acta Math. 187, 237 (2001); Acta Math. 187, 275 (2001); Ann. Inst. Henri Poincaré PR 38, 109 (2002).

[10] J. Cardy, J. Phys. A: Math. Gen. 36, L379 (2003); arXiv: math-ph/0301039

[11] B. Duplantier, Phys. Rev. Lett. 82, 3940 (1999); arXiv: cond-mat/9901008 Phys. Rev. Lett. 84, 1363 (2000); arXiv: cond-mat/9908314 